\title{
OPEN Comparing effects of intraoperative fluid and vasopressor infusion on intestinal microcirculation
}

\author{
Chia-Ning Fan ${ }^{1}$, Szu-Jen Yang ${ }^{1}$, Po-Yuan Shih ${ }^{1}$, Ming-Jiuh Wang ${ }^{1}$, Shou-Zen Fan ${ }^{1}$, \\ Jui-Chang Tsai ${ }^{2}$, Wei-Zen Sun ${ }^{1}$, Chih-Min Liu ${ }^{1,3 \otimes}$ \& Yu-Chang Yeh ${ }^{1,3 凶}$
}

Several studies have revealed that vasopressor may be more appropriate for treating intraoperative hypotension and preventing hypervolemia. This study compared the effects of vasopressor infusion and fluid supplementation on intestinal microcirculation during treating intraoperative hypotension. Thirty-two rats were randomly divided into the following four groups: Light Anesthesia group (LA, $0.8-1 \%$ isoflurane); Deep Anesthesia group (DA, 1.5-1.8\% isoflurane); Fluid DA group (1.5-1.8\% isoflurane and fluid supplementation); and Norepinephrine DA group (1.5-1.8\% isoflurane and norepinephrine infusion). At 240 min, perfused small vessel density (PSVD) of the mucosa did not differ significantly between the Fluid DA and Norepinephrine DA groups [26.2 (3.2) vs 28.9 (2.5) mm/ $\left.\mathrm{mm}^{2}, P=0.077\right]$, and tissue oxygen saturation of the mucosa was lower in the Fluid DA groups than in the Norepinephrine DA groups [ 48 (7) vs 57 (6) \%, $P=0.02$ ]. At $240 \mathrm{~min}$, TSVD and PSVD of the seromuscular layer were higher in the Norepinephrine DA group than in the Fluid DA group. Fluid administration was higher in the Fluid DA group than in the Norepinephrine DA group [66 (25) vs. 9 (5) $\mu \mathrm{L} / \mathrm{g}, P=0.001]$. Our results showed that norepinephrine can resuscitate intraoperative hypotension related microcirculatory alteration and avoid fluid overload.

Perioperative tissue hypoperfusion may result in postoperative complications and increased mortality ${ }^{1}$. The common causes of perioperative hypoperfusion are general anesthesia-induced hypotension, blood loss, and fluid loss. Intraoperative administration of crystalloids is commonly used to treat perioperative hypoperfusion. However, research has proved that fluid loading has no influence on anesthesia-induced hypotension ${ }^{2}$, and excessive fluid supplementation may cause hypervolemia-related glycocalyx impairment ${ }^{3}$, tissue edema, respiratory distress syndrome ${ }^{4}$, and abdominal compartment syndrome ${ }^{5}$. Postoperative fluid overload has been demonstrated to be strongly correlated with mortality ${ }^{6}$. Several studies have shown that vasopressors may be more appropriate for treating anesthesia-induced hypotension and prevent hypervolemia ${ }^{7,8}$. Furthermore, microcirculatory dysfunction can occur in the absence of arterial hypotension ${ }^{9-12}$. Intestinal microcirculatory dysfunction can lead to the disruption of the intestinal mucosal barrier and the development of multiple organ dysfunction syndrome ${ }^{13}$. To the best of our knowledge, no study has compared the treatment effects of fluid supplementation and vasopressor infusion on intestinal microcirculation. The primary aim of this study was to compare the treatment effects of fluid supplementation and vasopressor infusion on intestinal microcirculation during intraoperative anesthesiarelated hypotension in an open abdominal surgery rat model (Fig. 1).

\section{Results}

Characteristics, treatments, and systemic hemodynamics. The mean body weights and standard deviation of the rats were 266 (8) $\mathrm{g}$ in the light anesthesia (LA) group, 265 (21) g in the deep anesthesia (DA) group, 253 (23) g in the Fluid DA group, and 266 (13) g in the Norepinephrine DA group. According to the treatment protocol, the mean total fluid supplementation and standard deviation for $240 \mathrm{~min}$ was $8 \mu \mathrm{l} / \mathrm{g}$ in the LA and DA groups. Fluid supplementation was higher in the Fluid DA group than in the Norepinephrine DA group [66 (25) vs. 9 (5) $\mu \mathrm{L} / \mathrm{g}, P=0.001$ ]. The mean requirement and standard deviation of norepinephrine for $240 \mathrm{~min}$ was 40 (11) mcg in the Norepinephrine DA group. Mean arterial pressure (MAP) and the heart rate are given in Fig. 2. In the DA group, isoflurane induced $14 \%$ and $21 \%$ decreases in MAP at $60 \mathrm{~min}$ and $240 \mathrm{~min}$, respectively,

\footnotetext{
${ }^{1}$ Department of Anesthesiology, National Taiwan University Hospital, Chung Shan S. Rd, No.7, Taipei 10002, Taiwan. ${ }^{2}$ Institute of Medical Device and Imaging, College of Medicine, National Taiwan University, Taipei, Taiwan. ${ }^{3}$ These authors contributed equally: Chih-Min Liu and Yu-Chang Yeh. ${ }^{\square}$ email: liu.chihmin@gmail.com; tonyyeh@ntuh.gov.tw
} 


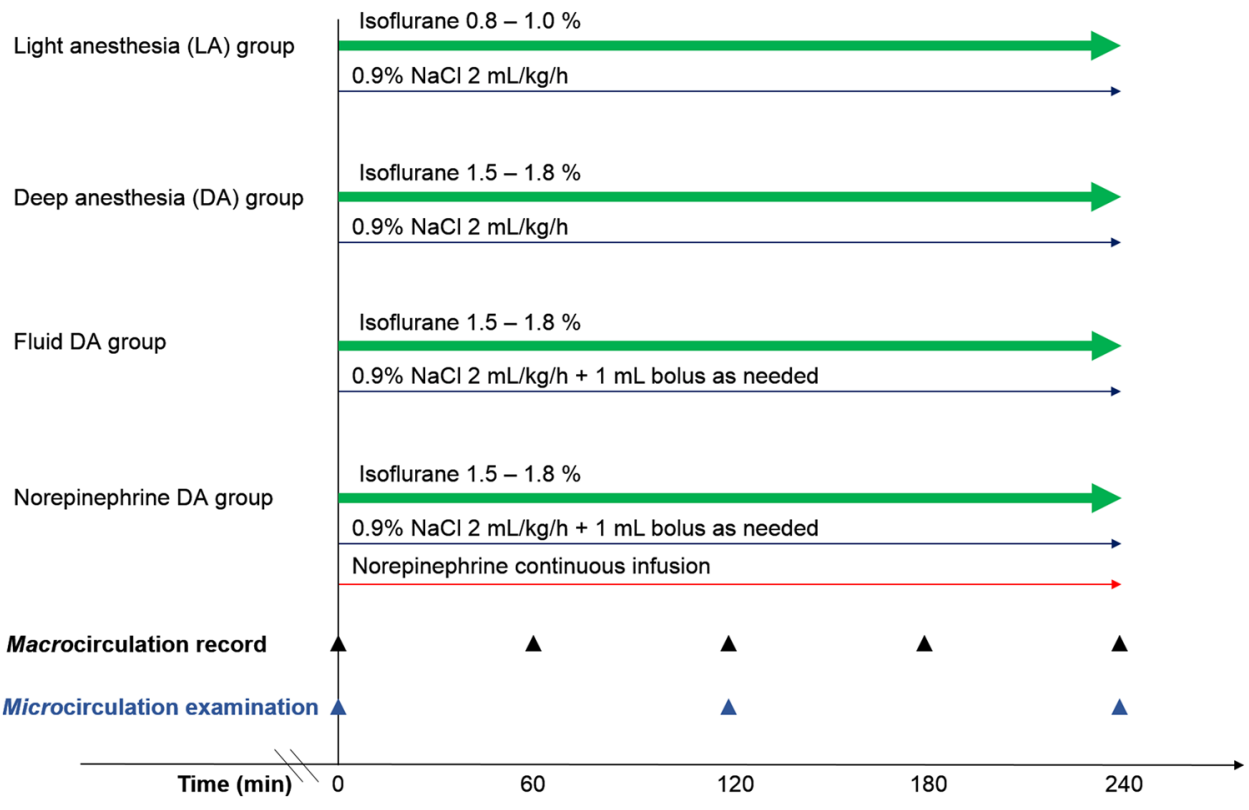

Figure 1. Grouping and treatment and examination protocol. The therapeutic goal of hypotension was to maintain a mean arterial pressure decreased by less than $10 \mathrm{~mm} \mathrm{Hg}$ of the initial value.
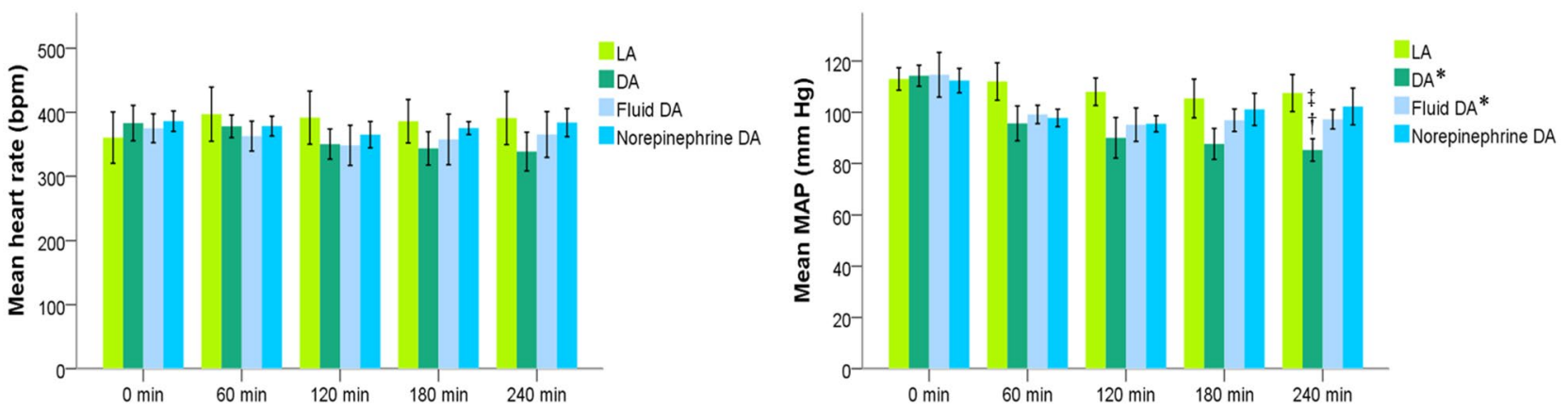

Figure 2. Heart rate and Mean arterial pressure (MAP) of the 4 groups. DA deep anesthesia, LA light anesthesia. ${ }^{\star} P<0.05$ vs. the LA group using repeated measures. At $240 \mathrm{~min},{ }^{\dagger} P<0.05$ vs. the LA group and ${ }^{\ddagger} P<0.05$ vs. the Norepinephrine DA group using one-way ANOVA with the Tukey test.

compared with the LA group. In repeated measures analysis, MAP was lower in the DA and Fluid DA groups than in the LA group. At $240 \mathrm{~min}$, heart rate did not significantly differ between the Fluid DA and Norepinephrine DA groups [384 (26) vs 365 (43) beats per minute, $P=0.788$ ]. At $240 \mathrm{~min}$, MAP did not significantly differ between the Fluid DA and Norepinephrine DA groups [102 (9) vs 97 (5) mm Hg, $P=0.493$ ].

Intestinal microcirculation of the mucosa. The microcirculation images of the mucosa and seromuscular layer at baseline and $240 \mathrm{~min}$ are shown in Fig. 3. For primary outcome comparison at $240 \mathrm{~min}$, perfused small vessel density (PVSD) of the mucosa did not differ significantly between the Fluid DA and Norepinephrine DA groups [26.2 (3.2) vs $28.9(2.5) \mathrm{mm} / \mathrm{mm}^{2}, P=0.077$ ]. Results of other exploratory variables are as follows. In repeated measures analysis, PSVD of the mucosa was lower in the DA group than in the LA and Norepinephrine DA groups (Fig. 4), but PSVD of the mucosa did not significantly differ between the DA and Fluid DA groups. Total small vessel density (TSVD) did not significantly differ among the four groups. At $240 \mathrm{~min}$, TSVD and PSVD of the mucosa in the Norepinephrine DA group were higher than those in the DA group [TSVD 29.0 (2.5) vs $24.7(2) \mathrm{mm} / \mathrm{mm}^{2}, P=0.011$; PSVD $28.9(2.5)$ vs $22.3(4.2) \mathrm{mm} / \mathrm{mm}^{2}, P=0.001$ ]. At $240 \mathrm{~min}$, TSVD and PVSD of the mucosa did not differ significantly between the DA and Fluid DA groups [TSVD: 24.7 (2.0) vs 26.4 (3.2) $\mathrm{mm} / \mathrm{mm}^{2}, P=0.545$; PSVD: 22.3 (4.2) vs $26.2(3.2) \mathrm{mm} / \mathrm{mm}^{2}, P=0.083$ ]. At $240 \mathrm{~min}$, TSVD of the mucosa did not differ significantly between the Fluid DA and Norepinephrine DA groups [26.4 (3.2) vs $29.0(2.5) \mathrm{mm} / \mathrm{mm}^{2}$, $P=0.195]$. In repeated measures analysis, $\mathrm{StO}_{2}$ of the mucosa was lower in the DA and Fluid DA groups than in the LA and Norepinephrine DA groups (Fig. 4). At $240 \mathrm{~min}$, tissue oxygen saturation $\left(\mathrm{StO}_{2}\right)$ of the mucosa was lower in the Fluid DA groups than in the Norepinephrine DA groups [48 (7) vs 57 (6) $\%, P=0.02$ ]. 

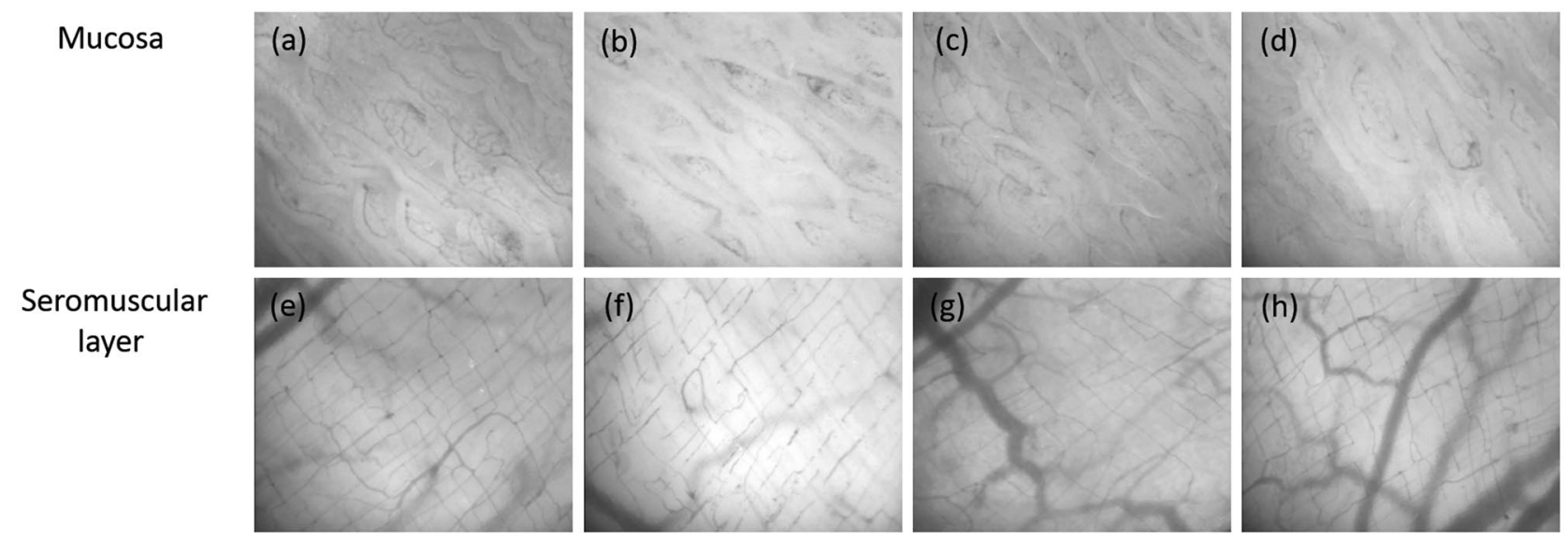

Figure 3. Microcirculation images of the terminal ileal mucosa and seromuscular layer. Microcirculation images of mucosa are shown in LA group (a), DA group (b), fluid DA group (c), and Norepinephrine DA group (d) at $240 \mathrm{~min}$. Microcirculation images of muscular layer are shown in LA group (e), DA group (f), Fluid DA group (g), and Norepinephrine DA group (h) at $240 \mathrm{~min}$. In the DA group, perfused small vessels were fewer in the intestinal mucosa (b) and seromuscular layer (f). Norepinephrine infusion could restore microcirculation in the intestinal mucosa (d). DA deep anesthesia, $L A$ light anesthesia.
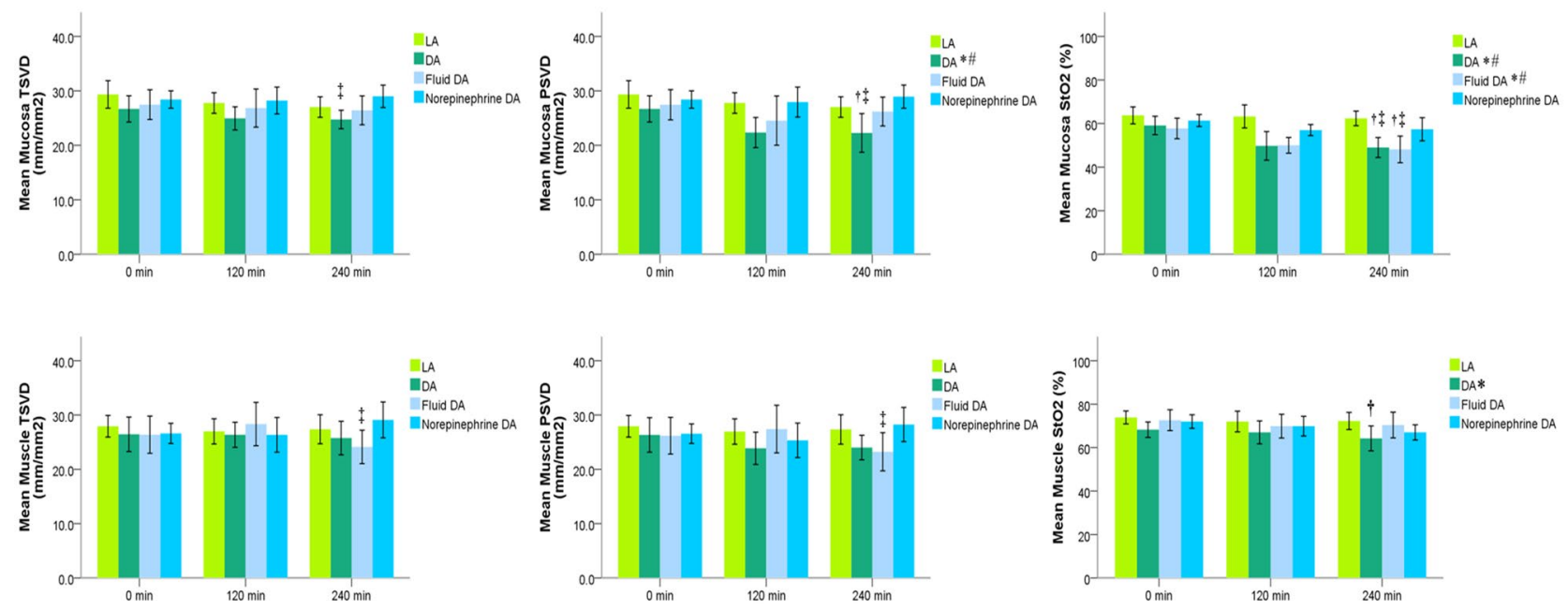

Figure 4. Microcirculatory parameters and tissue oxygen saturation of the terminal ileal mucosa and seromuscular layer. Primary outcome was compared using t test, and PVSD of the mucosa did not differ significantly between the Fluid DA and Norepinephrine DA groups [26.2 (3.2) vs $28.9(2.5) \mathrm{mm} / \mathrm{mm}^{2}$, $P=0.077]$. DA deep anesthesia, $L A$ light anesthesia, $P S V D$ perfused small vessel density, $S_{2} \mathrm{O}_{2}$ tissue oxygen saturation, TSVD total small vessel density. ${ }^{\star} P<0.05$ vs. the LA group and ${ }^{\#} P<0.05$ vs. the Norepinephrine DA group using repeated measures. At $240 \mathrm{~min},{ }^{\dagger} P<0.05$ vs. the LA group and ${ }^{\ddagger} P<0.05$ vs. the Norepinephrine DA group using one-way ANOVA with the Tukey test.

Intestinal microcirculation of the seromuscular layer. In repeated measures analysis, TSVD and PSVD of the seromuscular layer did not differ significantly among the four groups (Fig. 4). At 240 min, TSVD and PSVD of the seromuscular layer were higher in the Norepinephrine DA group than in the Fluid DA group [TSVD: $29.1(4.0)$ vs $24.1(3.7) \mathrm{mm} / \mathrm{mm}^{2}, P=0.05$; PSVD: 28.3 (3.8) vs $23.2(4.2) \mathrm{mm} / \mathrm{mm}^{2}, P=0.039$ ]. In repeated measures analysis, $\mathrm{StO}_{2}$ of the seromuscular layer was lower in the DA group than in the LA groups (Fig. 4). At $240 \mathrm{~min}, \mathrm{StO}_{2}$ of the seromuscular layer was lower in the DA group than in the LA groups [72 (5) \% vs 64 (7) \%, $P=0.045]$, but $\mathrm{StO}_{2}$ of the seromuscular layer did not significantly differ among the DA, Fluid DA, and Norepinephrine DA groups. 


\section{Discussion}

Our study did not show a significant difference of PSVD of the intestinal mucosa at 240 min between norepinephrine infusion and fluid administration. From the results of comparisons of other exploratory variables, we found that norepinephrine improve tissue oxygenation of the intestinal mucosa and the microcirculation of the terminal seromuscular layer with less amount of fluid when compared with fluid administration.

Our results that norepinephrine infusion resulted in better intestinal microcirculation than fluid supplementation may indicate that norepinephrine can be safely used to restore intraoperative anesthesia-related hypotension. This finding is supported by the animal study of Hiltebrand et al. ${ }^{14}$, in which low to moderate doses of norepinephrine were found to preserve hemodynamic stability without any major concerns related to splanchnic organ perfusion and oxygenation. Norepinephrine actively expel blood from the splanchnic capacitance vessels, causing a rapid increase in the venous return. Pathophysiologic mechanisms of anesthesia-related hypotension include vasodilation, impairment of sympathetic nervous system, and compromised baroreflex regulation ${ }^{15}$. Norepinephrine can attenuate vasodilation and decrease the requirement of fluid supplement. Our results support that the adequate use of vasopressor can significantly decrease the amount of fluid administered. Over-administration of saline can lead to hyperchloremic metabolic acidosis and can damage the endothelial glycocalyx ${ }^{16}$. In addition, administrating a large volume of crystalloids to maintain normovolemia can reduced capillary perfusion and tissue oxygen partial pressure ${ }^{17}$. Aggressive fluid therapy leads to increased cardiac filling pressures, which results in the release of natriuretic peptides, and these peptides markedly disrupt the glycocalyx structure and function, leading to increased vascular leakage ${ }^{18}$. Moreover, the resuscitation effect of fluid supplementation may be limited or not persistent. Norberg et al. reported that a bolus of $25 \mathrm{~mL} / \mathrm{kg}$ saline administered to healthy volunteers during isoflurane anesthesia had no effect on decreased blood pressure ${ }^{2}$.

A relevant concern is that difference in microcirculatory alterations between the mucosa and seromuscular layer. In the present study, compared with seromuscular layer, microcirculation was more severely affected in the mucosa during intraoperative hypotension. In a septic shock pig model, Hiltebrand et al. suggested that decreased capillary density may cause intestinal mucosal acidosis ${ }^{19}$. Mucosal microcirculatory dysfunction may account for the development of intestinal edema, stress ulcer bleeding, and bacterial translocation. Conversely, microcirculatory dysfunction was more severe in the seromuscular layer than in the mucosa during fluid supplementation for intraoperative hypotension. We suggested that overloaded fluid may result in more tissue edema in the seromuscular layer than in the mucosa. Hydrostatic edema has been reported to activate the signal transduction pathway that results in intestinal contractile dysfunction ${ }^{20}$. Microcirculatory dysfunction in the seromuscular layer may account for ileus.

Coherence of the macrocirculation and the microcirculation is the correlation between systemic hemodynamic variables and microcirculatory variables ${ }^{21}$. If hemodynamic coherence exists, correcting systemic hemodynamic variables is able to correct microcirculatory perfusion and oxygen delivery ${ }^{21}$. However, many studies have described conditions of a loss of hemodynamic coherence between macrocirculation and microcirculation ${ }^{22,23}$. We then did a post-hoc investigation of the correlation among heart rate, MAP, TSVD, PSVD, and StO $\mathrm{S}_{2}$ at $240 \mathrm{~min}$. We found that MAP was weakly correlated with PSVD (correlation coefficient $=0.363, P=0.041)$ and $\mathrm{StO}_{2}$ (correlation coefficient $=0.530, P=0.002$ ). Further studies are warranted to investigate the coherence of microcirculation variables and other systemic hemodynamic variables (eg. cardiac output, urine output) or laboratory data (eg. lactate level).

This study has several limitations. First, in the Fluid DA group, the MAP goal could not be maintained even with aggressive volume resuscitation. This observation is compatible with the result of Norberg et al. ${ }^{2}$. Second, the anesthetized rats were under spontaneous breathing. Mechanical ventilation with considerably positive endexpiratory pressure can lead to splanchnic hypoperfusion and marked decreases in hepatic, portal venous and mesenteric blood flow ${ }^{24}$. Third, our model was an open abdominal surgery model. Further studies in rat model with an intact abdominal wall, the effect of increase intra-abdominal pressure resulted from excessive intestinal edema should be considered. Fourth, as our previous description of the hyperchloremic metabolic acidosis after over-administration of saline, it is warranted for further studies to investigate the treatment effect of a balanced fluid on microcirculation.

In conclusion, norepinephrine can resuscitate intraoperative hypotension related microcirculatory alteration and avoid fluid overload. Moreover, overload fluid may impair microcirculation in the intestinal seromuscular layer. We suggest that the resuscitating effects on microcirculation should be considered in further studies of investigating perioperative hypotensive resuscitation.

\section{Methods}

Animals. In this study, 32 male Wistar rats (body weight $250 \pm 50$ g; Biolasco Taiwan Co., Taipei, Taiwan) were used, and this study was approved by the Animal Care and Use Committee of the Laboratory Animal Center of the College of Medicine, National Taiwan University, Taipei, Taiwan (No. 20130287). The rats were maintained on a 12-h light-dark cycle with free access to water and food. The rats were handled according to the Guidelines for the Treatment of Laboratory Animals of the Institute Animal Care and Use Committee of National Taiwan University.

Grouping and treatment protocol. The 32 rats were randomly assigned to four groups ( 8 rats in each group) (Fig. 1). In the LA group, the rats received $0.8-1 \%$ (the inspiratory concentration) isoflurane inhalation, with a continuous infusion of $0.9 \%$ sodium chloride at $2 \mathrm{~mL} / \mathrm{kg} / \mathrm{h}$ as the maintenance fluid supplementation through an external jugular vein catheter. In the DA group, the rats received 1.5-1.8\% isoflurane inhalation with a continuous infusion of $0.9 \%$ sodium chloride at $2 \mathrm{~mL} / \mathrm{kg} / \mathrm{h}$. In the Fluid DA group, rats receiving $1.5-1.8 \%$ isoflurane inhalation with a continuous infusion of $0.9 \%$ sodium chloride, and the infusion rate was adjusted to 
maintain MAP decreased by less than $10 \mathrm{~mm} \mathrm{Hg}$ of the initial value. The rats were given a $1 \mathrm{~mL}$ bolus of $0.9 \%$ sodium chloride for persistent hypotension. In the Norepinephrine DA group, rats received 1.5\%- $1.8 \%$ isoflurane inhalation with a continuous intravenous infusion of norepinephrine ( $60 \mathrm{mcg} / \mathrm{mL}$ in $5 \%$ dextrose), and the infusion rate was adjusted to maintain MAP decreased by less than $10 \mathrm{mmHg}$ of the initial value. Subsequently, they were administered a bolus of $1 \mathrm{~mL}$ of $0.9 \%$ sodium chloride for persistent hypotension. The infusion concentration of norepinephrine was determined by our pilot study.

Anesthesia and surgical procedure. Anesthesia, tracheostomy, and right common carotid artery and external jugular vein cannulation were performed as described in our previous study ${ }^{25}$. Atropine sulfate $(0.05 \mathrm{mg} / \mathrm{kg}$ in $0.9 \%$ sodium chloride) was administered subcutaneously to reduce respiratory tract secretions and block vagal reflexes elicited by the manipulation of the intestinal viscera. Arterial blood pressure and the heart rate were continually monitored via the right common carotid artery catheter and recorded at baseline, $60 \mathrm{~min}, 120 \mathrm{~min}, 180 \mathrm{~min}$, and $240 \mathrm{~min}$. The rectal temperature was continuously monitored. A $3 \mathrm{~cm} \mathrm{long}$ midline laparotomy was performed to exteriorize a segment of terminal ileum which was located at 6 to $10 \mathrm{~cm}$ proximal to the ileocecal valve. The mucosa and seromuscular layer of the terminal ileum were prepared for microcirculation examination as described in our previous study ${ }^{26}$. Euthanasia was performed by exsanguination cardiac arrest under anesthesia after finishing all examinations.

Measurement of intestinal microcirculation and tissue oxygen saturation. After a 15-min stabilization period, baseline microcirculation examinations for the mucosa and seromuscular layer was performed with a sidestream dark-field video microscope (MicroScan, Microvision Medical, Amsterdam, The Netherlands) and a tissue oxygen monitor (white light reflectance spectroscopy, moorVMS-OXY, Moor Instruments Ltd., Devon, UK). The time after the examination was set as $0 \mathrm{~min}$, and the rats received treatment according to their grouping. Intestinal microcirculation was examined again at $120 \mathrm{~min}$ and $240 \mathrm{~min}$.

Intestinal microcirculation parameters, including TSVD (less than $20 \mu \mathrm{m}$ ) and PSVD were analyzed using semi-automated analysis software (AVA 3.0, Academic Medical Center, University of Amsterdam, Amsterdam, Netherlands) according to the guidelines of microcirculation analysis ${ }^{27,28}$. At each time point, three continuous image sequences $(10 \mathrm{~s}$ ) were digitally stored for each measured site, and the data of the three images were averaged for obtaining the required statistics. Analyses were performed by a single investigator who was blinded to grouping. At each time point, $\mathrm{StO}_{2}$ was measured at three points of the intestinal mucosa and seromuscular layer, and the data of these points were averaged for statistical calculation.

Primary outcome and sample size calculation. Primary outcome in this study was defined as the difference of PSVD of the mucosa between the Fluid DA and Norepinephrine DA group. Based on our experience ${ }^{26,29}$, this study ( $n=8$ rats per group) was powered to detect a $10 \%$ difference of PSVD of the mucosa between the two groups at $240 \mathrm{~min}$, with an $\alpha$ level of 0.05 (single-tailed) and a $\beta$ level of 0.2 (80\% power), assuming a control PSVD of $29(2.3) \mathrm{mm} / \mathrm{mm}^{2}$.

Statistical analysis. Data analysis was performed using statistical software (SPSS 20; IBM SPSS, USA). Data normality was examined by W/S test ${ }^{30}$, and appropriate statistical analysis was used according to the normality of data. Primary outcome was examined by the Mann-Whitney U-test or the t-test according to the normality of PSVD. A $P$ value of $<0.05$ was considered significant. Other exploratory variables were examined as follows. Differences in means over time among the four groups were analyzed using general linear model repeated measures ANOVA (Analysis of Variance). Differences in means at $240 \mathrm{~min}$ among the four groups were analyzed using one-way ANOVA, followed by the Tukey test for post hoc analysis. The $P$ values for comparisons of exploratory data were not adjusted.

\section{Data availability}

The datasets generated during and/or analysed during the current study are available from the corresponding author on reasonable request.

Received: 26 July 2020; Accepted: 27 October 2020

Published online: 16 November 2020

\section{References}

1. Parker, T., Brealey, D., Dyson, A. \& Singer, M. Optimising organ perfusion in the high-risk surgical and critical care patient: a narrative review. Br. J. Anaesth. 123, 170-176. https://doi.org/10.1016/j.bja.2019.03.027 (2019).

2. Norberg, A. et al. Population volume kinetics predicts retention of $0.9 \%$ saline infused in awake and isoflurane-anesthetized volunteers. Anesthesiology 107, 24-32. https://doi.org/10.1097/01.anes.0000268387.34758.6d (2007).

3. Bruegger, D. et al. Atrial natriuretic peptide induces shedding of endothelial glycocalyx in coronary vascular bed of guinea pig hearts. Am. J. Respir. Crit. Care Med. 289, H1993-1999. https://doi.org/10.1152/ajpheart.00218.2005 (2005).

4. National Heart, L. et al. Comparison of two fluid-management strategies in acute lung injury. N. Engl. J. Med. 354, 2564-2575. https://doi.org/10.1056/NEJMoa062200 (2006).

5. O'Mara, M. S., Slater, H., Goldfarb, I. W. \& Caushaj, P. F. A prospective, randomized evaluation of intra-abdominal pressures with crystalloid and colloid resuscitation in burn patients. J. Trauma. 58, 1011-1018 (2005).

6. Lowell, J. A., Schifferdecker, C., Driscoll, D. F., Benotti, P. N. \& Bistrian, B. R. Postoperative fluid overload: not a benign problem. Crit. Care Med. 18, 728-733 (1990).

7. Turek, Z., Sykora, R., Matejovic, M. \& Cerny, V. Anesthesia and the microcirculation. Semin. Cardiothorac. Vasc. Anesth. 13, 249-258. https://doi.org/10.1177/1089253209353134 (2009). 
8. Jacob, M., Chappell, D., Conzen, P., Finsterer, U. \& Rehm, M. Blood volume is normal after pre-operative overnight fasting. Acta Anaesthesiol. Scand. 52, 522-529. https://doi.org/10.1111/j.1399-6576.2008.01587.x (2008).

9. Farquhar, I. et al. Decreased capillary density in vivo in bowel mucosa of rats with normotensive sepsis. J. Surg. Res. 61, 190-196. https://doi.org/10.1006/jsre.1996.0103 (1996).

10. Lam, C., Tyml, K., Martin, C. \& Sibbald, W. Microvascular perfusion is impaired in a rat model of normotensive sepsis. J. Clin. Investig. 94, 2077-2083. https://doi.org/10.1172/JCI117562 (1994).

11. De Backer, D., Creteur, J., Preiser, J. C., Dubois, M. J. \& Vincent, J. L. Microvascular blood flow is altered in patients with sepsis. Am. J. Respir. Crit. Care Med. 166, 98-104 (2002).

12. Sakr, Y., Dubois, M. J., De Backer, D., Creteur, J. \& Vincent, J. L. Persistent microcirculatory alterations are associated with organ failure and death in patients with septic shock. Crit. Care Med. 32, 1825-1831 (2004).

13. Leaphart, C. L. \& Tepas, J. J. 3rd. The gut is a motor of organ system dysfunction. Surgery 141, 563-569. https://doi.org/10.1016/j. surg.2007.01.021 (2007).

14. Hiltebrand, L. B., Koepfli, E., Kimberger, O., Sigurdsson, G. H. \& Brandt, S. Hypotension during fluid-restricted abdominal surgery: effects of norepinephrine treatment on regional and microcirculatory blood flow in the intestinal tract. Anesthesiology 114, 557-564. https://doi.org/10.1097/ALN.0b013e31820bfc81 (2011).

15. Kouz, K., Hoppe, P., Briesenick, L. \& Saugel, B. Intraoperative hypotension: pathophysiology, clinical relevance, and therapeutic approaches. Indian J. Anaesth. 64, 90-96. https://doi.org/10.4103/ija.IJA_939_19 (2020).

16. Torres, L. N., Chung, K. K., Salgado, C. L., Dubick, M. A. \& Torres Filho, I. P. Low-volume resuscitation with normal saline is associated with microvascular endothelial dysfunction after hemorrhage in rats, compared to colloids and balanced crystalloids. Crit. Care 21, 160. https://doi.org/10.1186/s13054-017-1745-7 (2017).

17. Funk, W. \& Baldinger, V. Microcirculatory perfusion during volume therapy: a comparative study using crystalloid or colloid in awake animals. Anesthesiology 82, 975-982. https://doi.org/10.1097/00000542-199504000-00022 (1995).

18. Woodcock, T. E. \& Woodcock, T. M. Revised Starling equation and the glycocalyx model of transvascular fluid exchange: an improved paradigm for prescribing intravenous fluid therapy. Br. J. Anaesth. 108, 384-394. https://doi.org/10.1093/bja/aer515 (2012).

19. Hiltebrand, L. B., Krejci, V., tenHoevel, M. E., Banic, A. \& Sigurdsson, G. H. Redistribution of microcirculatory blood flow within the intestinal wall during sepsis and general anesthesia. Anesthesiology 98, 658-669. https://doi.org/10.1097/00000542-20030 3000-00014 (2003).

20. Shah, S. K. et al. A murine model for the study of edema induced intestinal contractile dysfunction. Neurogastroenterol. Motil. 22, 1132-e1290. https://doi.org/10.1111/j.1365-2982.2010.01546.x (2010).

21. Ince, C. Hemodynamic coherence and the rationale for monitoring the microcirculation. Crit. Care 19(Suppl 3), S8. doi:https:// doi.org/10.1186/cc14726 (2015).

22. De Backer, D. et al. Microcirculatory alterations in patients with severe sepsis: impact of time of assessment and relationship with outcome. Crit. Care Med. 41, 791-799. https://doi.org/10.1097/CCM.0b013e3182742e8b (2013).

23. Tachon, G. et al. Microcirculatory alterations in traumatic hemorrhagic shock. Crit. Care Med. 42, 1433-1441. https://doi. org/10.1097/CCM.0000000000000223 (2014).

24. Fujita, Y. Effects of PEEP on splanchnic hemodynamics and blood volume. Acta Anaesthesiol. Scand 37, 427-431. https://doi. org/10.1111/j.1399-6576.1993.tb03742.x (1993).

25. Yeh, Y. C. et al. Effects of eritoran tetrasodium, a toll-like receptor 4 antagonist, on intestinal microcirculation in endotoxemic rats. Shock 37, 556-561. https://doi.org/10.1097/SHK.0b013e31824e20ef (2012).

26. Yeh, Y. C. et al. Dexmedetomidine prevents alterations of intestinal microcirculation that are induced by surgical stress and pain in a novel rat model. Anesth. Analg 115, 46-53. https://doi.org/10.1213/ANE.0b013e318253631c (2012).

27. De Backer, D. et al. How to evaluate the microcirculation: report of a round table conference. Crit. Care 11, R101. https://doi. org/10.1186/cc6118 (2007)

28. Ince, C. et al. Second consensus on the assessment of sublingual microcirculation in critically ill patients: results from a task force of the European Society of Intensive Care Medicine. Intensive Care Med. 44, 281-299. https://doi.org/10.1007/s00134-018-5070-7 (2018).

29. Yeh, Y. C. et al. Enoxaparin sodium prevents intestinal microcirculatory dysfunction in endotoxemic rats. Crit Care 16, R59. https ://doi.org/10.1186/cc11303 (2012).

30. Singh, A. S. \& Masuku, M. Normality and data transformation for applied statistical analysis. Int. J. Econ. Commerce Manag. 2, 1-11. https://citeseerx.ist.psu.edu/viewdoc/download?doi=10.1.1.679.9264\&rep=rep1\&type=pdf (2014).

\section{Acknowledgements}

We thank the advices and technical support from members of National Taiwan University Hospital Center of Microcirculation Medical Research (NCMMR). We thank Roger Lien (Technician, MicroStar Instruments CO., Ltd., Taipei, Taiwan) for their technical assistances in microcirculation examination. This manuscript was edited by Wallace Academic Editing.

\section{Author contributions}

C.M.L. and Y.C.Y. designed the study. C.M.L. and Y.C.Y. conducted the study. C.N.F, S.J. Y., P.Y.S, and Y.C.Y. analyzed the data. C.N.F, C.M.L., and Y.C.Y. wrote the main manuscript. C.N.F. and Y.C.Y. prepared the figures. P.Y.S., M.J.W, S.Z.F., W.Z.S., J.C.T., C.M.L., and Y.C.Y. revised the manuscript. All authors reviewed the manuscript.

\section{Funding}

This work was partly supported by research grants from the National Taiwan University Hospital (NTUH 104-S2629).

\section{Competing interests}

The authors declare no competing interests.

\section{Additional information}

Correspondence and requests for materials should be addressed to C.-M.L. or Y.-C.Y.

Reprints and permissions information is available at www.nature.com/reprints.

Publisher's note Springer Nature remains neutral with regard to jurisdictional claims in published maps and institutional affiliations. 
(c) (i) Open Access This article is licensed under a Creative Commons Attribution 4.0 International cc) License, which permits use, sharing, adaptation, distribution and reproduction in any medium or format, as long as you give appropriate credit to the original author(s) and the source, provide a link to the Creative Commons licence, and indicate if changes were made. The images or other third party material in this article are included in the article's Creative Commons licence, unless indicated otherwise in a credit line to the material. If material is not included in the article's Creative Commons licence and your intended use is not permitted by statutory regulation or exceeds the permitted use, you will need to obtain permission directly from the copyright holder. To view a copy of this licence, visit http://creativecommons.org/licenses/by/4.0/.

(C) The Author(s) 2020 